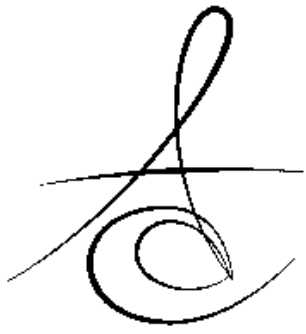

\title{
KÖK KANALININ APİKALİNDE YAPAY OLARAK STANDARDİZE EDİLMİş OLUKTAN KALSİYUM HİDROKSİTIN UZAKLAŞTIRILMASINDA QMİX SOLÜSYONUNUN ETKİNLİĞİ
}

\section{EFFICACY OF QMIX IN REMOVAL OF CALCIUM HYDROXIDE FROM ARTIFICIAL STANDARDIZED GROOVE IN APICAL ROOT CANAL}

\author{
Dr.Dt. Salih DÜZGÜN* \\ Dr.Dt. Firdevs AKPEK ${ }^{* *}$ \\ Dt. Özge ULUSAN**
}

\author{
Doç.Dr. Hüseyin Sinan TOPÇUOĞLU** \\ Dr. Dt. Gamze TOPÇUOĞLU*** \\ Dt. Ahmet AKTI**
}

Makale Kodu/Article code: 2860

Makale Gönderilme tarihi: 22.05.2016

Kabul Tarihi: 01.07.2016

\section{ÖZET}

$\mathrm{Bu}$ çalışmanın amacı kök kanalının apikalinde yapay olarak standardize edilmiş oluklardan kalsiyum hidroksitin (KH) uzaklaştırılmasında QMix 2 in 1 solüsyonunun etkinliğini değerlendirmektir. 118 adet çekilmiş tek köklü üst kanin dişi ProTaper sisteminin F5 boyutundaki eğesine kadar genişletildi. Bu dişler uzunlamasına olarak 2 'ye ayrıldı ve her bir parçanın apikal kısmında standardize edilmiş oluk hazırlandı. Sonrasında bu oluklar distile su ile kalsiyum hidroksit tozunun karıştırılmasıyla elde edilen pat ile dolduruldu ve bu dişler yeniden birleştirildi. KH'i uzaklaştırmak için dişler aşağıdaki irrigasyon protokolüne göre, rastgele 2 kontrol ve 6 deneysel grubuna ayrıldı. (1) Konvansiyonel şırınga irrigasyonu (KŞİ)/Sodyum Hipoklorit $(\mathrm{NaOCl})+E$ tilendiamin Tetraasetik Asit (EDTA) , (2) QMix/KŞİ, (3) NaOCl+ QMİX /KŞİ, (4) $\mathrm{NaOCl}+\mathrm{EDTA}$ /Pasif Ultrasonik İrrigasyon (PUİ), (5) QMix/PUİ ve (6) QMix+NaOCl/PUİ. Oluklarda kalan KH miktarı 4 dereceli skorlama sistemi kullanılarak 25 büyütmede stereomikroskop altında değerlendirildi. Veriler Kruskal-Wallis ve Mann-Whitney U istatistiksel testleri kullanılarak değerlendirildi. Yapay standardize edilmiş oluklardaki kalan $\mathrm{KH}$ miktarı tüm deneysel gruplarda gözlendi. PUI/QMix+NaOCl ve $\mathrm{PUI} / \mathrm{NaOCl}+\mathrm{EDTA}$ diğer gruplara göre $\mathrm{KH}^{\prime} \mathrm{i}$ uzaklaştırmada daha etkiliydi $(P<0.05)$; fakat, $\mathrm{PUI} / \mathrm{QMix}+\mathrm{NaOCl}$ ve PUİ/NaOCl+EDTA grupları arasında istatistiksel olarak anlamlı fark bulunmadı ( $P$ $>0.05$ ). Diğer dört deneysel grup arasında $\mathrm{KH}^{\prime} \mathrm{i}$ uzaklaştırma bakımından istatistiksel olarak fark bulunmadı ( $P$ > 0.05). İrrigasyon tekniklerinin hiçbirisi, kök kanalının apikalindeki yapay standardize edilmiş oluktan $\mathrm{KH}^{\prime} \mathrm{i}$ tam olarak uzaklaştıramadı.

Anahtar kelimeler: Kalsiyum Hidroksit, İrrigasyon, $\mathrm{NaOCl}$, QMix, Ultrasonik

\section{ABSTRACT}

The aim of this study was to evaluate the efficacy of QMix in the removal of calcium hydroxide $(\mathrm{CH})$ from an artificial standardized groove in the apical root canal. One hundred and eighteen extracted singlerooted canine teeth were prepared using ProTaper system instruments up to size F5. The roots were split longitudinally, and a standardized groove was prepared in the apical part of one segment. The grooves were then filled with $\mathrm{CH}$ powder mixed with distilled water, and the roots were reassembled. The roots were randomly divided into 6 experimental groups and 2 control groups, according to the following irrigation methods: (1) conventional syringe irrigation (CSI) with sodium hypochlorite $(\mathrm{NaOCl})+$ ethylenediaminetetraacetic acid (EDTA), (2) CSI with QMix, (3) CSI with QMix + $\mathrm{NaOCl}$, (4) passive ultrasonic irrigation (PUI) with $\mathrm{NaOCl}+$ EDTA, (5) PUI with QMix, and (6) PUI with QMix + NaOCl. The amount of remaining medicament was evaluated under a stereomicroscope at $25 \times$ magnification using a 4-grade scoring system. Data were evaluated using the Kruskal-Wallis and Mann-Whitney $U$ tests. Remnants of $\mathrm{CH}$ were found in all experimental groups. $\mathrm{PUI} / \mathrm{QMix}+\mathrm{NaOCl}$ and $\mathrm{PUI} / \mathrm{NaOCl}+\mathrm{EDTA}$ were superior to the other groups $(p<0.05)$; however, there was no significant difference between the PUI/QMix + $\mathrm{NaOCl}$ and PUI/NaOCl + EDTA groups $(p>0.05)$. There were no significant differences between the other four experimental groups ( $p>$ 0.05). None of the irrigation techniques completely removed $\mathrm{CH}$ from an artificial standardized groove in an apical root canal.

Key words: Calcium hydroxide, Irrigation, $\mathrm{NaOCl}$, QMix, Root canal, Ultrasonic

\footnotetext{
* Adana Fatma Kemal Timuçin Ağız Ve Diş Sağlığı Hastanesi

** Erciyes Üniversitesi, Diş Hekimliği Fakültesi, Endodonti AD

${ }^{* * *}$ Erciyes Üniversitesi Diş Hekimliği Fakültesi, Pedodonti AD.
} 


\section{GİRİŞ}

Kök kanal sisteminin dezenfeksiyonu için kanal içi ilaç kullanımı kök kanal tedavisinin başarısını arttırır. Kanal içi ilaçlar öncelikli olarak kök kanal sistemi içerisindeki bakterilerinin azaltılması, elimine edilmesi ve seanslar arasında bakteri çoğalmasının engellenmesi için kullanılır. ${ }^{1,2}$ Kalsiyum Hidroksit (KH) antienflamatuar, organik doku çözme kapasitesi ve antimikrobiyal potansiyelinden dolayı en sıklıkla kullanılan kanal içi ilaçtır., ${ }^{3,4} \mathrm{KH}$ kök kanal dolgusundan önce kök kanalından uzaklaştırımalı çünkü kök kanal duvarları üzerindeki $\mathrm{KH}$ kalıntıları kök kanal dolgusunun kalitesini olumsuz etkileyebilir.5,6 $\mathrm{KH}$ kaldırılmasında en sıklıkla tanımlanan metot Sodyum Hipoklorit ( $\mathrm{NaOCl}$ ) ve Etilendiamin Tetraasetik Asit (EDTA) solüsyonlarının kullanımıyla birlikte ana apikal eğenin çalışma boyunda kullanılmasıdır. ${ }^{7-9}$ Sonik ve ultrasonik cihazları, döner enstrümanları ve kanal fırçalarını içeren farklı metotlar $\mathrm{KH}$ kanal içi ilacının kaldırılmasında önerilmektedir. Fakat, bu metotların hiçbirisinin, özellikle kök kanalının apikal üçlüsündeki KH'i tamamen uzaklaştıramadığı bir çok araştırmada ortaya konmuştur. ${ }^{10-12}$

QMix 2in1 (Dentsply Tulsa Dental, Tulsa, OK) (Resim 1) yüksek antimikrobiyal aktivite gösteren ve smear tabakasının kaldırılmasında etkili olan yeni bir endodontik irrigasyon solüsyonudur. Bu solüsyon, EDTA, Klorheksidin,deterjan ve su içerir. QMix 'in kimyasal dizaynı, klorheksidinin EDTA ve $\mathrm{NaOCl}$ ile kombine olarak kullanıldığı zaman meydana gelen çökelti oluşumunu engellemektedir. ${ }^{13} \mathrm{Bu}$ çalışmanın amacı kökün apikal üçlüsünde yapay olarak standardize edilmiş oluktan $\mathrm{KH}^{\prime}$ in kaldırımasında farklı irrigasyon tekniklerinin etkinliğini değerlendirmektir.

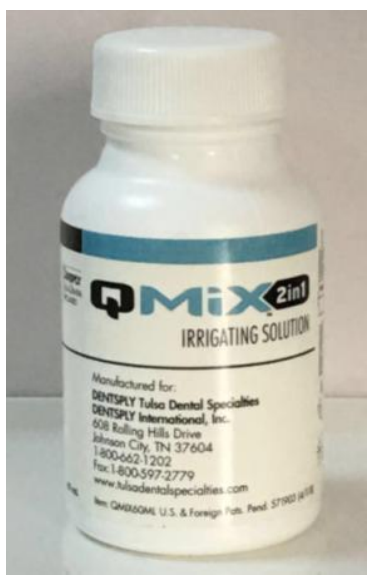

Resim 1. Çalışmada kullanılan irrigasyon solüsyonu (QMix)

\section{GEREÇ VE YÖNTEM}

118 adet, mine-sement birleşiminden kök apeksine kadar ölçülmüş benzer kök boylarına sahip olan, çekilmiş insan tek köklü üst kanin dişleri bu çalışma için seçildi. Tek kök kanallı, çürük, çatlak ve kırığı olmayan, iç ve dış rezorpsiyon ve kalsifikasyon belirtisi göstermeyen, tamamlanmış kök ucuna sahip, Schneider' $\mathrm{e}^{14}$ göre $<5^{\circ}$ eğime sahip dişler çalışmaya dahil edildi. Kalsifikasyon, rezorpsiyon, önceden yapılmış kök kanal tedavisi olmayan tek kanallı dişlerin varlığını doğrulamak için bukkolingual ve meziodistal açılardan deney öncesi radyografiler alındı. Dişler deney başlangıcına kadar distile suda saklanmıştır. Standardizasyonu elde etmek için seçilen dişlerin kronları her bir dişin son boyu $23 \mathrm{~mm}$ olacak şekilde aşındırıldı. Endodontik giriş kaviteleri su soğutması altında yüksek hızlı bir aerotöre takılmış elmas frez (Diatech, Coltene Whaledent, Altstätten, Switzerland) kullanılarak hazırlandı. 15 numaralı $\mathrm{K}$ tipi eğe apikal foramenden görülünceye kadar kanal içerisinde ilerletildi ve bu boy ölçüldü. Bu ölçümden $1 \mathrm{~mm}$ çıkartılarak çalışma boyu (ÇB) belirlendi. 118 adet kök ana apikal eğe F5 (\#50, 0.05 açılı) oluncaya kadar ProTaper (Dentsply Maillefer) döner alet sistemi kullanılarak şekillendirildi. Şekillendirme sırasında, kök kanalları, her bir eğe değişikliği arasında 29 ebadındaki endodontik iğne (NaviTip; Ultradent, South Jordan, UT, USA) ve şırınga kullanılarak 3ml \% 2,5 lik NaOCl ile yıkanmıştır. Genişletme tamamladıktan sonra, kök kanalları 1 dakika süreyle $5 \mathrm{~mL} \% 17$ EDTA ve bir daki- ka süreyle $5 \mathrm{~mL} \% 3 \mathrm{NaOCl}$ irrigasyon solüsyonları ile yıkanmıştır.Son olarak kök kanalları $10 \mathrm{~mL}$ distile su ile yıkandı ve steril kurutma kağıtları ile kurulandı.

Kök kanal genişletmesini takiben, dişler silikon ölçü maddesi ile modifiye Eppendorf tüplerinde (ColtenelWhaledent AG, Switzerland) sabitlendi. Ölçü maddesi kaldırıldıktan sonra, kök kanal içerisine penetrasyon engellenerek bol su irrigasyonu altında tüm dişlerin bukkal ve lingual yüzeyleri üzerine elmas disk ile oluklar oluşturuldu. Sonrasında bu dişler küçük keski ile 2 yarım parçaya ayrıldı. Apikal yarıda enstrümente edilmemiş kanal genişlemelerini taklit etmek için , apeksten 2-6 mm uzaklıkta $4 \mathrm{~mm}$ uzunluğunda , $0,2 \mathrm{~mm}$ genişliğinde ve $0,5 \mathrm{~mm}$ derinliğinde standart oluklar oluşturuldu. ${ }^{15} 5$ diş negatif kontol grubu olarak belirlendi ve bu dişe $\mathrm{KH}$ materyali yerleştirilmedi. $\mathrm{KH}$ tozu distile su ile karıştırılarak hazırlanan karışım, bir 
plugger yardımıyla kalan 113 dişin apikalindeki olukların içerisine yerleştirildi. Sonrasında yarıya ayrılmış dişler yeniden birleştirildi ve eppendorf tüpleri içerisinde yerleştirildi. Giriş kaviteleri geçici dolgu materyali ile kapatıldı (Cavit; 3M ESPE, Seefeld, Germany). Dişler, $\% 100$ nemlilikte, $37^{\circ} \mathrm{C}$ de 7 gün süresince saklandı. 113 örneğin 5 tanesi pozitif kontrol grubu olarak belirlendi ve bu dişlere $\mathrm{KH}$ kaldırma prosedürü uygulanmadı.

\section{KH kaldırılması ve deneysel gruplar}

Dış kök yüzeyleri, apikal foramen boyunca herhangi bir irrigasyon solüsyonunun taşmasını engellemek için tırnak ojesi ile kapatıldı. Geçici dolgu maddesi kaldırıldıktan sonra, örnekler aşağıda belirtildiği gibi 6 deneysel gruba ayrılmıştır.

Grup 1: KŞİ/ $5 \mathrm{~mL} \mathrm{NaOCl} \mathrm{+} 5$ mL EDTA (n = 18)

Oluklardan $\mathrm{KH}$ kaldırıması için, çalışma boyundan $1 \mathrm{~mm}$ kısa yerleştirilmiş 29 ebadındaki iğne ve şırınga kullanılarak (NaviTip; Ultradent, South Jordan, UT, USA) $5 \mathrm{~mL} \% 17$ 'lik EDTA solüsyonu ve bunu takiben $5 \mathrm{~mL} \% 3^{\prime}$ lük $\mathrm{NaOCl}$ solüsyonuyla ile irrigasyon yapıldı.

\section{Grup 2: KŞİ/QMix $(n=18)$}

$\mathrm{Bu}$ grupta çalışma boyundan $1 \mathrm{~mm}$ kısa yerleştirilmiş 29 ebadındaki iğne ve şırınga kullanılarak (NaviTip) $10 \mathrm{~mL}$ QMix 2in1 solüsyonu ile irrigasyon yapıldı.

\section{Grup 3: KŞİ/QMix + NaOCl (n = 18)}

$\mathrm{Bu}$ grupta çalışma boyundan $1 \mathrm{~mm}$ kısa yerleştirilmiş 29 ebadındaki iğne ve şırınga kullanılarak (NaviTip) $5 \mathrm{~mL} \%$ 3'lük $\mathrm{NaOCl}$ solüsyonunu takiben 5 $\mathrm{mL}$ QMix 2in1 solüsyonuyla irrigasyon yapıldı.

\section{Grup 4: $\mathbf{P U I ̇} / \mathbf{N a O C l}+$ EDTA $(\mathbf{n}=18)$}

Bu grupta, $5 \mathrm{~mL} \%$ 3'lük\% $\mathrm{NaOCl}$ ve $5 \mathrm{~mL}$ \%17'lik EDTA solüsyonlarının her birisi ultrasonik cihaz (EMS, Le Sentier, Switzerland) kullanılarak pasif olarak ajite edildi. 15 boyutunda ve 0.02 açılı düz ultrasonik uç (ESI instrument), ucun serbest olarak titreşimine izin verecek şekilde, çalışma boyundan $1 \mathrm{~mm}$ kısa olacak şekilde kanal içinde konumlandırıldı. Ultrasonik uç, her bir irrigasyon solüsyonu için, 1 dakika süresince 6 güç ayarında aktive edildi.

\section{Grup 5: PUİ/QMix (n = 18)}

Bu grupta, $10 \mathrm{~mL}$ QMix 2in1 irrigasyon solüsyonu olarak kullanıldı. İrrigasyon solüsyonun ajitasyonu Grup 4'te uygulanan yöntemle aynı olacak şekilde 2 dakika olarak yapıldı.

\section{Grup 6: PUI/QMix + NaOCl $(\mathbf{n}=18)$}

$\mathrm{KH}$ kaldırılmasında, $5 \mathrm{~mL}$ QMix 2in1 ve $5 \mathrm{~mL} \%$ 3'lük $\mathrm{NaOCl}$ irrigasyon solüsyonları uygulandı. İrrigasyon solüsyonlarının ajitasyonları önceki gruplardaki (Grup 4 ve 5) ile aynı şekilde uygulandı. Her bir irrigasyon solüsyonu için ajitasyon zamanı 1 dakikaydı.

İrrigasyon prosedürü sonrası, kanallar kağıt kurutucular kullanılarak kurulandı ve kökler yeniden 2' ye ayrıldı. Dijital görüntüler stereomikroskoba (BX60; Olympus) bağlı dijital kamera (DP-70; Olympus, Tokyo, Japan) kullanılarak 25 büyütme altında alındı ve bilgisayara aktarıldı. 2 kalibre edilmiş gözlemci, birbirinden habersiz olarak van der Sluis ${ }^{16}$ tarafından tanımlanan skorlama sistemini kullanarak oluklarda kalan KH miktarını skorladı. Skorlama sistemi aşağıdaki gibiydi:

$0=$ oluk boş, $1=$ oluğun yarısından daha azı $\mathrm{KH}$ ile dolu, 2=oluğun yarısından fazlası $\mathrm{KH}$ ile dolu, 3= oluk tamamen $\mathrm{KH}$ ile dolu (Resim 2).
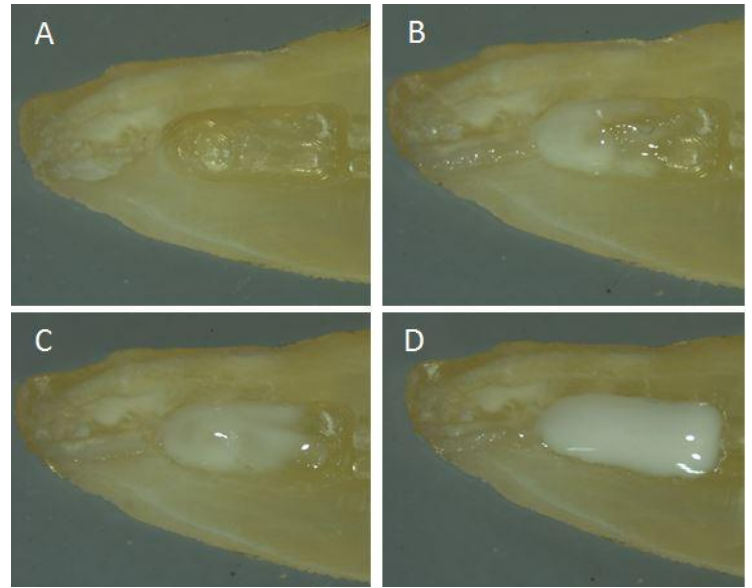

Resim 2. van der Sluis tarafından tanımlanan 4 dereceli skorlama sistemi

Görüntüler, birey içi tekrarlanabilirliği değerlendirmek için 7 gün sonra 2 kalibre edilmiş gözlemci tarafından yeniden değerlendirildi. Gözlemciler arası uyumu analiz etmek için Kappa testi uygulandı. Normalite içi uygulanan Kolmogorov-Smirnov testi veri dağııımının normal olarak dağılıp dağılmadığını açığa çıkarttı. Gruplar arasındaki farklılıklar, Kruskal Wallis ve Mann-Whitney $U$ testleri kullanılarak analiz edildi. İstatistiksel analizler, SPSS 16.0 yazılımı (SPSS Inc., Chicago, IL, USA) kullanılarak gerçekleştirildi. Anlamlılık seviyesi $p<0.05$. olarak belirlendi. 


\section{BULGULAR}

Kappa testi, yapay standardize edilmiş oluklaran $\mathrm{KH}$ kaldırılmasında gözlemciler arası uyumun \%96,2 olduğunu gösterdi. Gözlemciler arası birey içi tekrarlanabilirliği \%97,4 (115/118) ve \% 96,6 ( 114/ 118 ) idi. Tablo 1 , oluklar içerisindeki kalan $\mathrm{KH}^{\prime}$ in gruplara göre miktarını göstermektedir. Deneysel grupların tümü pozitif ve negatif kontrol gruplarından istatistiksel olarak farklıydı ( $p<0.05$ ). Deneysel grupların hiçbirisi yapay standardize edilmiş oluktan $\mathrm{KH}$ 'i tamamen kaldıramadı. Deneysel gruplar arasındaki $\mathrm{KH}$ kaldırma sonuçları arasında istatistiksel olarak belirgin farlılıklar vardı ( $p<0.05)$. PUİ/QMix + NaOCl ve PUİ/ $\mathrm{NaOCl}+$ EDTA grupları arasında istatistiksel olarak anlamlı farklılık bulunmadı ( $p>0.05$ ). İlave olarak, $\mathrm{KŞİ} / \mathrm{NaOCl}+$ EDTA,KŞİ/QMix,KŞİ/QMix + $\mathrm{NaOCl}$, and PUI/QMix grupları arasında da istatistiksel farklılık tespit edilmedi ( $p>0.05$ ).

Tablo 1. Farklı irrigasyon rejimleri sonrasında her bir skor değerindeki örneklerin yüzdesi ve sayısı

\begin{tabular}{lccc} 
& & & Skor \\
\hline Grup & $\boldsymbol{n}$ & 0 & 1 \\
\hline Negatif Kontrol & 5 & $5(100 \%)$ & $0(0 \%)$ \\
Pozitif Kontrol & 5 & $0(0 \%)$ & $0(0 \%)$ \\
KŞİ/NaOCl+EDTA & 18 & $4(22.2 \%)$ & $3(16.6 \%)$ \\
KŞİ/QMix & 18 & $3(16.6 \%)$ & $2(11.1 \%)$ \\
KŞİ/QMix+NaOCl & 18 & $4(22.2 \%)$ & $3(16.6 \%)$ \\
PUİ/NaOCl+EDTA & 18 & $10(55.5 \%)$ & $7(38.8 \%)$ \\
PUİ/QMix & 18 & $3(16.6 \%)$ & $6(33.3 \%)$ \\
PUİ/QMix+NaOCl & 18 & $9(50 \%)$ & $8(44.4 \%)$ \\
\hline
\end{tabular}

n; örneklerin sayıSı, KŞİ; konvansiyonel şııınga irrigasyonu, PUİ pasif ultrasonik irrigasyon

\section{TARTIŞMA}

Çalışmamızın dizaynındaki oluk modelinin avantajı, olukların lokasyonu ve standardize edilmiş boyutlarının iyi bir gözlemciler arası uyum ve yüksek birey içi tekrarlanabilirlikle standardize bir değerlendirmeye izin vermesidir. Bu dizaynın en büyük dezavantajı ise, yapay standardize edilmiş olukların doğal kök kanal sisteminin karmaşıklığını temsil edememesidir. Bu yüzden, in vivo oval kanal içi düzensizliklerden ve isthmuslardansa yapay oluklardan $\mathrm{KH}$ kaldırıması daha kolay olabilir.
Salin solüsyonu, distile su, $\mathrm{NaOCl}$, EDTA, sitrik asit ve bunların kombinasyonu $\mathrm{KH}^{\prime}$ in kök kanalından uzaklaştırılması için kullanılmıştır. Bu solüsyonların hiç birisinin $\mathrm{KH}$ 'i tamamen kaldıramadığı iyi bilinmektedir. ${ }^{17}$ Bu çalışmada, yapay standardize edilmiş oluklardan $\mathrm{KH}$ kaldırılmasında yeni bir irrigasyon solüsyonu olan QMix'in etkinliğini değerlendirildi. Önceki çalışmalar, NaOCl'nin kalsiyum gibi inorganik materyallerin çözülmesindeki sınırlı yeteneğinden dolayı kök kanallarından $\mathrm{KH}^{\prime}$ in kaldırılmasında tek başına yeterli olmadığını gösterdi.9,10 Bu yüzden, çalışmamız sadece $\mathrm{NaOCl}$ 'nin olduğu bir grubu içermemektedir. Klorheksidin solüsyonunu takiben $\mathrm{NaOCl}$ ile yapılan irrigasyonda dentin tübüllerinde para kloranilin (PKA) çökeltisi oluştuğu bilinmektedir. ${ }^{18}$ Fakat, Kolosowski ve ark. ${ }^{19}$ QMix solüsyonunu takiben $\mathrm{NaOCl}$ ile yapılan irrigasyon sonrasında dentin tübüllerinde PKA veya başka çökeltilere rastlanmadığını göstermiştir. Şimdiki çalışmada, yapay standardize edilmiş oluklardan $\mathrm{KH}$ kaldırmak için, $\mathrm{NaOCl}$ solüsyonu, QMix ve EDTA solüsyonunun kombinasyonu ile kullanıldı.

Çalışmamızın bulguları, PUİ/QMix + $\mathrm{NaOCl}$ ve $\mathrm{PUİ} / \mathrm{NaOCl}+$ EDTA gruplarının test edilen diğer irrigasyon prosedürlerine göre daha fazla $\mathrm{KH}^{\prime} \mathrm{i}$ uzaklaştırdığını göstermiştir. Çapar ve ark. ${ }^{20}$ taklit edilmiş kök kanal düzensizliklerinden $\mathrm{KH}^{\prime}$ in kaldırımasında çeşitli irrigasyon tekniklerinin etkinliklerini karşılaştırmışlar ve kök kanalının apikal üçlüsündeki yapay standardize edilmiş oluktan $\mathrm{KH}^{\prime}$ in kaldırılmasında $\mathrm{PUI} / \mathrm{NaOCl}+$ EDTA irrigasyon tekniğini $\mathrm{KŞI} / \mathrm{NaOCl}$ + EDTA irrigasyon tekniğinden daha etkili olduğunu göstermişlerdir.Bu bulgu çalışmamızın bulgularıyla uyumludur. Rödig ve ark. ${ }^{8}$ tarafından yapılan bir çalışmada, $\mathrm{KH}^{\prime} \mathrm{in}$ kaldırılmasında $\mathrm{NaOCl}$ ve EDTA kombinasyonu sadece EDTA solüsyonundan belirgin olarak daha az etkili bulunmuştur. $\mathrm{NaOCl}$ solüsyonunun $\mathrm{KH}$ kaldırılmasını belirgin olarak arttırmadığını da belirtmişlerdir. Bu durum, $\mathrm{NaOCl}$ solüsyonunun inorganik doku çözmedeki sınırlı yeteneğiyle ilişkilendirilebilir. Bu bulguya zıt olarak, Çalt ve Serper ${ }^{6}, \mathrm{NaOCl}$ irrigasyonunu takiben EDTA irrigasyonunun $\mathrm{KH}$ 'in tamamen kaldırıldığını göstermiştir. Bizim çalışmamızda, PUİ içeren ve içermeyen $\mathrm{NaOCl}+$ EDTA grupları ve PUİ içeren ve içermeyen $\mathrm{NaOCl}+$ QMix ( QMix solüsyonu EDTA içerir) grupları yapay olarak standardize edilmiş oluklardan $\mathrm{KH}$ i tamamen uzaklaştıramadı. Bu durum, kullanılan deneysel metotlardaki farklılıklardan dolayı olabilir. Çalışmamızdan farklı olarak ta, Çalt ve Serper ${ }^{6}$ kalan

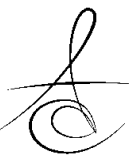


KH miktarını değerlendirmek için bütün kanal duvarlarını değerlendirmişlerdir.

PUİ'nin irrigasyon solüsyonunu ajite edici etkisi akustik akış ve kavitasyon prensibine dayanır. Oluşturulan mikroakışlardan dolayı, irrigasyon solüsyonun Şırınga ile dağıtımıyla karşılaştırıldığında PUİ kök kanal sisteminden daha fazla dentin debrisi kaldırabilir. ${ }^{21}$ Muhtemelen, KŞİ ile karşılaştırıldığında PUİ sırasında $\mathrm{KH}$ 'in daha etkili kaldırılmasında benzer mekanizmalar etkili olmuş olabilir. PUİ tarafından oluşturulan irrigasyon akışının daha yüksek hızı, kök kanallarından $\mathrm{KH}^{\prime} \mathrm{in}$ kaldırılmasında onun etkinliğini açıklayabilir. ${ }^{22}$

\section{SONUÇLAR}

Test edilen hiçbir irrigasyon tekniği kök kanaIının apikalindeki yapay standardize edilmiş oluktan $\mathrm{KH}$ 'i tamamen kaldıramadı. $\mathrm{Bu}$ in vitro çalışmanın sınırları dahilinde, PUİ/QMix + NaOCl grubunun PUİ/EDTA + $\mathrm{NaOCl}$ grubu kadar $\mathrm{KH}^{\prime}$ in kaldırılmasında etkili olduğu sonucuna varılabilir.

\section{KAYNAKLAR}

1. Siqueira JF Jr, de Uzeda M. Influence of different vehicles on the antibacterial effects of calcium hydroxide. J Endod 1998;24:663-5.

2. Law A, Messer H. An evidence-based analysis of the antibacterial effectiveness of intracanal medicaments. J Endod 2004;30:689-94.

3. Mohammadi Z, Dummer PM. Properties and applications of calcium hydroxide in endodontics and dental traumatology. Int Endod J 2011; 44:697-730.

4. Farhad A, Mohammadi Z. Calcium hydroxide: a review. Int Dent J 2005;55:293-301.

5. Böttcher DE, Hirai VH, Da Silva Neto UX, Grecca FS. Effect of calcium hydroxide dressing on the long-term sealing ability of two different endodontic sealers: an in vitro study. Oral Surg Oral Med Oral Pathol Oral Radiol Endod 2010;110:386-9.

6. Calt S, Serper A. Dentinal tubule penetration of root canal sealers after root canal dressing with calcium hydroxide. J Endod 1999;25:431-3.
7. Balvedi RP, Versiani MA, Manna FF, Biffi JC. A comparison of two techniques for the removal of calcium hydroxide from root canals. Int Endod J 2010;43:763-8.

8. Rödig T, Vogel S, Zapf A, Hülsmann M. Efficacy of different irrigants in the removal of calcium hydroxide from root canals. Int Endod J 2010;43:519-27.

9. Salgado RJ, Moura-Netto C, Yamazaki AK, Cardoso LN, de Moura AA, Prokopowitsch I. Comparison of different irrigants on calcium hydroxide medication removal: microscopic cleanliness evaluation. Oral Surg Oral Med Oral Pathol Oral Radiol Endod 2009;107:580-4.

10. Kenee DM, Allemang JD, Johnson JD, Hellstein J, Nichol BK. A quantitative assessment of efficacy of various calcium hydroxide removal techniques. J Endod 2006;32:563-5.

11. Alturaiki $S$, Lamphon $H$, Edrees $H$, Ahlquist $M$. Efficacy of 3 different irrigation systems on removal of calcium hydroxide from the root canal: a scanning electron microscopic study. J Endod 2015;41:97-101.

12. Taşdemir T, Celik D, Er K, Yildirim T, Ceyhanli $K T$, Yeşilyurt C. Efficacy of several techniques for the removal of calcium hydroxide medicament from root canals. Int Endod J 2011;44:505-9.

13. Bui TB, Baumgartner JC, Mitchell JC. Evaluation of the interaction between sodium hypochlorite and chlorhexidine gluconate and its effect on root dentin. J Endod 2008;34:181-5.

14. Schneider SW. A comparison of canal preparations in straight and curved root canals. Oral Surg Oral Med Oral Pathol 1971;32:271-5.

15. Lee SJ, Wu MK, Wesselink PR. The effectiveness of syringe irrigation and ultrasonics to remove debris from simulated irregularities within prepared root canal walls. Int Endod J 2004;37:672-8.

16. van der Sluis LW, Wu MK, Wesselink PR. The evaluation of removal of calcium hydroxide paste from an artificial standardized groove in the apical root canal using different irrigation methodologies. Int Endod J 2007;40:52-7.

17. Arslan H, Gok T, Saygili G, Altintop H, Akçay M, Çapar ID. Evaluation of effectiveness of various irrigating solutions on removal of calcium hydroxide mixed with $2 \%$ chlorhexidine gel and 
detection of orange-brown precipitate after removal. J Endod 2014;40:1820-3.

18. Basrani BR, Manek S, Mathers D, Fillery E, Sodhi RN. Determination of 4-chloroaniline and its derivatives formed in the interaction of sodium hypochlorite and chlorhexidine by using gas chromatography. J Endod 2010;36:312-4.

19. Kolosowski KP, Sodhi RN, Kishen A, Basrani BR. Qualitative analysis of precipitate formation on the surface and in the tubules of dentin irrigated with sodium hypochlorite and a final rinse of chlorhexidine or QMiX. J Endod 2014;40:203640.

20. Capar ID, Ozcan E, Arslan H, Ertas H, Aydinbelge HA. Effect of different final irrigation methods on the removal of calcium hydroxide from an artificial standardized groove in the apical third of root canals. J Endod 2014;40:451-4.

21. Lee SJ, Wu MK, Wesselink PR. The efficacy of ultrasonic irrigation to remove artificially placed dentine debris from different-sized simulated plastic root canals. Int Endod J 2004;37:607-12.

22. Jiang $L M$, Verhaagen $B$, Versluis $M$, van der Sluis LW. Influence of the oscillation direction of an ultrasonic file on the cleaning efficacy of passive ultrasonic irrigation. J Endod 2010;36:1372-6

\author{
Yazışma Adresi \\ Dr. Salih DÜZGÜN \\ Adana Fatma Kemal Timuçin \\ Ağız Ve Diş Sağlığı Hastanesi \\ ADANA \\ e-mail: salih_duzgun@hotmail.com
}

